\title{
Low-intensity body building exercise induced rhabdomyolysis: a
}

\section{case report}

Massimiliano Gagliano1, Daniela Corona1, Giuseppe Giuffrida1, Alessia Giaquinta ${ }^{1}$, Tiziano Tallarita1 ${ }^{1}$ Domenico Zerbo1, Massimiliano Sorbello ${ }^{2}$, Annalaura Paratore ${ }^{2}$, Carla Virgilio ${ }^{1}$, Alessandro Cappellani ${ }^{1}$, Pierfrancesco Veroux ${ }^{1}$ and Massimiliano Veroux ${ }^{* 1}$

\author{
Address: ${ }^{1}$ Department of Surgery, Transplantation and Advanced Technologies; Vascular Surgery and Organ Transplant Unit, University Hospital \\ of Catania, Via Santa Sofia, 8695123 Catania, Italy and ${ }^{2}$ Department of Surgery, Transplantation and Advanced Technologies; Anaesthesia and \\ Intensive Care Unit, University Hospital of Catania, Via Santa Sofia, 8695123 Catania, Italy \\ Email: Massimiliano Gagliano - veroux@unict.it; Daniela Corona - coronadany@libero.it; Giuseppe Giuffrida - veroux@unict.it; \\ Alessia Giaquinta - veroux@unict.it; Tiziano Tallarita - veroux@unict.it; Domenico Zerbo - veroux@unict.it; \\ Massimiliano Sorbello - maxsorbello@gmail.it; Annalaura Paratore - maxsorbello@gmail.it; Carla Virgilio - veroux@unict.it; \\ Alessandro Cappellani - veroux@unict.it; Pierfrancesco Veroux - veroux@unict.it; Massimiliano Veroux* - veroux@unict.it \\ * Corresponding author
}

Published: 5 January 2009

Cases Journal 2009, 2:7 doi:10.1186/1757-1626-2-7

This article is available from: http://www.casesjournal.com/content/2/I/7

(C) 2009 Gagliano et al; licensee BioMed Central Ltd.

This is an Open Access article distributed under the terms of the Creative Commons Attribution License (http://creativecommons.org/licenses/by/2.0), which permits unrestricted use, distribution, and reproduction in any medium, provided the original work is properly cited.
Received: 4 October 2008

Accepted: 5 January 2009

\begin{abstract}
Introduction: Rhabdomyolysis is a severe and debilitating condition that promotes muscle breakdown and is a relatively rare, not always diagnosed cause of acute renal failure (ARF) with an 8-20\% reported incidence. Exertional rhabdomyolysis only appears in adult patients $24-48 \mathrm{~h}$ after strenuous activities as military basic training, weight lifting, and marathon running.
\end{abstract}

Case presentation: A 30-year-old man was admitted to our department because of weakness and painful swelling of the muscles as well as dark urine appearing $24 \mathrm{~h}$ after carrying out a bodybuilding exercises of low intensity. The development of an acute exertional rhabdomyolysis was confirmed by the increased serum enzyme levels and myoglobinuria. The patient was treated with intravenous sodium chloride, and sodium bicarbonate. The nephrotoxicity of myoglobin was decreased by forced alkaline diuresis.

Conclusion: The reported case emphasizes the occurrence of acute rhabdomyolysis even in those who underwent a low-intensity exercise. A proper treatment is mandatory to avoid a sudden worsening of clinical conditions eventually evolving to acute renal failure.

\section{Introduction}

Rhabdomyolysis is a severe and debilitating condition that promotes muscle breakdown and is a relatively rare, not always diagnosed cause of acute renal failure (ARF) with an $8-20 \%$ reported incidence $[1,2]$. Exertional rhabdomyolysis only appears in adult patients $24-48 \mathrm{~h}$ after strenuous activities as military basic training, weight lifting, and marathon running [3]. Strenuous exercise can result in damage to skeletal muscle cells and in most cases is resolved without consequences. However, in case of severe damage, there is a huge release of myoglobin which, in high concentrations and under certain condi- 
tions, such as dehydration and heat stress, may precipitate in the kidneys, thereby resulting in ARF $[1,2,4]$. The classical clinical presentation of rhabdomyolysis is characterized by myalgias, muscle weakness, and darkened urine [4]. Laboratory results may include delayed high levels of muscle enzymes in the plasma (CPK, ALT, AST, LDH) myoglobinemia and myoglobinuria, disturbance in blood electrolyte balance, and in severe cases, disturbance in coagulatory function and DIC $[2,4]$. The main treatment for rhabdomyolysis is fluids administration and maintaining urination, in order to preserve renal function [4]. The treatment results and mortality rate were primarily influenced by the patients' general condition at admission. A favourable outcome may be obtained especially in those patients who were hospitalized in a nephrological center[2].

We report a unique case of acute rabdomyolysis induced by a low-intensity body building exercise.

\section{Case presentation}

A 30-year-old man was admitted to our department because of weakness and painful swelling of the muscles as well as dark urine appearing 24 hours after carrying out a body-building exercise of low intensity. The development of an "acute exertional rhabdomyolysis" was confirmed by the increased serum enzyme levels and myoglobinuria. Total serum creatine phosphokinase (CPK) was 70,962 IU/L (normal range (n.r) 35 - $175 \mathrm{IU} /$ L). The laboratory tests revealed a serum creatinine of 2.1 $\mathrm{mg} / \mathrm{dl}$ and the serum LDH level was 4,981 IU/L (n.r 250 $500 \mathrm{IU} / \mathrm{L}$ ) with an elevation of the serum ALT and AST levels of 347 IU/l (n.r 5 - 40 IU/L) and 1,205 IU/L (n.r 5 $42 \mathrm{IU} / \mathrm{L}$ ), respectively. A myoglobin serum concentration of $1,702 \mathrm{ng} / \mathrm{ml}$ (n.r $28-72 \mathrm{ng} / \mathrm{mL}$ ) was also found. A diagnosis of pigmenturia due to urinary myoglobin excretion was suspected, and the patients was immediately treated with $3000 \mathrm{ml}$ of intravenous sodium chloride, and $500 \mathrm{ml}$ of sodium bicarbonate. The nephrotoxicity of myoglobin was decreased by forced alkaline diuresis. The patient did well after two days, and CPK, AST and ALT values gradually restored after ten days.

\section{Discussion}

Cases of rhabdomyolysis associated with other forms of excessive physical exertion are commonly reported, however cases associated with low-intensity and weight lifting are very rarely reported, probably because most cases in this setting go unrecognized and are diagnosed as simple muscle strain [5]. The exact incidence of rhabdomyolysis in this setting of body building exercise remains unknown. The reported case emphasizes the occurrence of acute rhabdomyolysis even in those who underwent a low-intensity exercise. A proper treatment is mandatory to avoid a sudden worsening of clinical conditions eventually evolving to acute renal failure.

\section{Abbreviations}

AST: Aspartate Aminotransferase; ALT: Alanine AminoTransferase; CPK: Creatine Posphokinase; DIC: Disseminated Intravascular Coagulation; LDH: Lactic dehydrogenase

\section{Consent}

Written informed consent was obtained from the patient for publication of this case report. A copy of the written consent is available for review by the Editor-in-Chief of this journal.

\section{Competing interests}

The authors declare that they have no competing interests.

\section{Authors' contributions}

MG was a major contributor in writing the manuscript. DC interpreted the laboratory data. GG has been involved in the draft of the manuscript. AG interpreted the data regarding the follow up of the patient. TT interpreted the data regarding the follow up of the patient. DZ was responsible of the management of the patient. MS was responsible of intensive care management. AP was responsible of intensive care management. $\mathrm{CV}$ has been involved in the draft of the manuscript. AC has been involved in the draft of the manuscript. PV was responsible of the management of the patient. MV was a major contributor in writing the manuscript and has given the final approval to the manuscript.

\section{References}

I. Visweswaran P, Guntupalli J: Rhabdomyolisis. Crit Care Clin 1999, I5:4I5-428.

2. Sulowicz W, Walatek B, Sydor A, Ochmanski W, Milkowski A, Szymczakiewicz-Multanowska A, Szumilak D, Krasniak A, Lonak H: Acute renal failure in patients with rhabdomyolysis. Med Sci Monit 2002, 8(I):CR24-CR27.

3. Sayers SP, Clarkson PM: Exercise induced rhabdomyolysis. Curr Sports Med Rep 2002, I:59-60.

4. Shani Y, Heled Y, Moran DS: Exercise rhabdomyolysis. Aviat Space Environ Med 200I, 72:856.

5. Sharma N, Winpenny H, Heymann T: Exercise induced rhabdomyolysis: even the fit may suffer. Int J Clin Pract 1999, 53:476-477. 